\title{
Impact of Pharmaceutical Care on Self-Administration of Outpatient Low-Molecular-Weight Heparin Therapy
}

\author{
Seraina Mengiardi1 ${ }^{*}$, Dimitrios A. Tsakiris ${ }^{2}$, Viviane Molnar ${ }^{3}$, Urs Kohlhaas-Styk ${ }^{3}$, \\ Michael Mittag1, Stephan Kraehenbuehl ${ }^{4}$, Kurt E. Hersberger ${ }^{1}$ \\ ${ }^{1}$ Pharmaceutical Care Research Group, University of Basel, Basel, Switzerland \\ ${ }^{2}$ Division of Hematology, University Hospital Basel, Basel, Switzerland \\ ${ }^{3}$ Clinic for Orthopedic Surgery and Traumatology, Kantonsspital Baselland, Bruderholz, Switzerland \\ ${ }^{4}$ Division of Clinical Pharmacology and Toxicology, University Hospital Basel, Basel, Switzerland \\ Email: *seraina.mengiardi@unibas.ch
}

Received 5 February 2014; revised 17 March 2014; accepted 28 March 2014

Copyright (C) 2014 by authors and Scientific Research Publishing Inc.

This work is licensed under the Creative Commons Attribution International License (CC BY).

http://creativecommons.org/licenses/by/4.0/

(c) (i) Open Access

\section{Abstract}

Outpatient subcutaneous (s.c.) therapies are becoming more and more common in the treatment of different diseases. The effectiveness of community-pharmacy-based interventions in preventing problems that arise during s.c. self-injections of low-molecular-weight heparins (LMWH) is unknown. Our objective was to provide a standard operating procedure (SOP) for community pharmacists and to compare pharmaceutical vs. standard care in both clinical and daily life settings. We hypothesized that: pharmaceutical care results in improved adherence, safety, and satisfaction, and in fewer complications; the interventions used are feasible in daily life; and the results achieved in clinical and daily life settings are comparable. In the clinical setting (randomized controlled trial), patients were recruited sequentially in hospital wards; in the daily life setting (quasi-experimental design with a comparison group), recruitment took place in community pharmacies by pharmacists and trained master students during their internship. Interventions were offered according to patient needs. Data were collected by means of a monitored self-injection at home and structured questionnaire-based telephone interviews at the beginning and the end of the LMWH treatment. The main outcome measures were: scores to assess patient's skills; syringe count to assess adherence; and frequency, effectiveness, and patient's assessment of received interventions. The results show a median age of the 139 patients of 54 years. Interventions resulted in improved application quality $(p<0.01)$ and knowledge $(p=0.03)$. Oral instructions were pivotal for improving patients' application quality. We found no significant score differences between the intervention groups in the clinical and daily life settings. Patients' baseline skills were high, with the lowest score being 0.86 (score range $\mathbf{- 2 . 0 0}$ to +2.00 ). Adherence rate was high (95.8\%). In conclusion, our SOP for pharmacist interventions was of good quality, adequate, ap-

"Corresponding author. 
preciated, and feasible in daily life. Patients are capable of managing s.c. injection therapies if adequate assistance is provided.

\title{
Keywords
}

\author{
Low-Molecular-Weight Heparin; Outpatients; Subcutaneous Injections; Self Administration; \\ Pharmaceutical Care; Community Pharmacy; Switzerland
}

\section{Introduction}

The number of medications that cannot be taken orally but have to be administered subcutaneously (s.c.) is rising; such medications are used in the treatment of thromboembolism, diabetes, multiple sclerosis, arthritis, anemia, cancer, female infertility, hepatitis B and C, migraine, osteoporosis, and HIV. Different devices are used to deliver such medication: pre-filled syringes, pens, injectors, and vials/ampules, where preparation is needed before injection. Self-injections in an outpatient setting are encouraged to strengthen patient responsibility for her/his own disease management, grant greater independence, and reduce costs.

For prophylaxis and treatment of venous thromboembolisms (VTE), the use of low-molecular-weight heparins (LMWH) is well established [1]. Therapies are often initiated during a hospital stay or at discharge, followed by daily s.c. self-injections for a period of time varying from days to weeks or even longer. Because after discharge most patients visit a community pharmacy to fill their prescription, pharmacists play an important role in the continuity of care by assuring correct drug use over the prescribed time [2] [3]. The community pharmacist's conventional role of preparing and dispensing drugs is changing, and the provision of new pharmaceutical services is needed [3]. The influence of pharmaceutical care on asthma, dyslipidemia, hypertension, and diabetes has been investigated [4]-[10], but knowledge of the effectiveness of community-pharmacy-based interventions on problems in self-administering s.c. injection therapies is lacking.

Enhancement of adherence is a multilevel challenge that includes a combination of different interventions, such as patient education with oral and written instructions, monitoring, telephone follow-up, reminder systems, and use of patient-tailored care [11] [12]. It has been reported in the literature that problems with self-administering outpatient LMWH treatments are prevalent, diverse, and may concern the injection itself or handling of the injection device [13]-[15]. Previous interventional studies recruited 40 - 528 patients, and concentrated mainly on orthopedic patients from selected clinics or hospitals [16]-[20]. All patients received educational programs that included information about VTE and LMWH, instructions in the injection technique, performing their first self-injection in the presence of a medical professional, written information, or a video tape. A search of the literature failed to identify studies that were controlled, examined feasibility of the interventions in daily life, or objectively assessed each patient's injection technique in everyday life after hospital discharge (e.g. directly observed therapy, DOT, during a home visit).

Our aims of the study were: 1 ) to provide a standard operating procedure (SOP) for the first instruction in the s.c. injection technique given by a community pharmacist and the subsequent pharmaceutical care provided during outpatient therapy, and 2) to compare intensive pharmaceutical care vs. standard care both in a clinical setting (hospital wards under study conditions) and in a daily life setting (community pharmacies following their daily routine). We hypothesized that: 1) intensive pharmaceutical care for outpatients self-injecting LMWH results in improved adherence, safety, and satisfaction, as well as in fewer complications, 2) the interventions used are feasible in the everyday routine of community pharmacies, and 3) the results achieved in clinical and daily life settings are comparable.

\section{Method}

\subsection{Setting and Study Population}

This study comprised both clinical and daily life settings. The clinical setting arm (ClinS) was a randomised controlled trial (RCT). Patients were recruited sequentially into the intervention (ClinS-I) or control (ClinS-C) group by the primary investigator from two orthopedic clinics (Kantonsspital Baselland, Bruderholz; University 
Hospital Basel), from an orthopedic early rehabilitation ward of the University Hospital Basel (Felix Platter-Spital), and from an emergency department (University Hospital Basel) between June 2007 and June 2009. The primary investigator had attended a certified course for parenteral injection techniques and four specialised courses on the s.c. injection technique, including clinical training by nursing staff.

The daily life setting arm (DailyS) was a quasi-experimental design with a comparison group. Patients were recruited sequentially in community pharmacies: for the control group (DailyS-C) by 65 trained students from the University of Basel during their internship between January and May 2008 ( $n=65$ community pharmacies) [14] and for the intervention group (DailyS-I) by trained community pharmacists between March 2008 and June 2009. We invited all community pharmacies in the region to attend one of our courses, which included background information and clinical training in the s.c. injection technique. Out of the course participants, 21 community pharmacies agreed to recruit patients for the DailyS-I arm. Community pharmacies recruiting for the DailyS-C arm were not allowed to recruit for the DailyS-I arm and contrariwise. We defined the following inclusion criteria: patients aged $\geq 18$ years with a prescription for an outpatient LMWH treatment with pre-filled syringes; Fragmin ${ }^{\circledR}$ (dalteparin; ClinS) or all brands of LMWH (DailyS); self-injection; prophylactic or therapeutic use; first or previous outpatient s.c. treatment; all therapy durations; no comprehension difficulties due to language.

\subsection{Interventions}

Our SOP comprised different interventions, which were offered and applied according to patient need (Table 1). The leaflet ( 4 pages) and the laminated manual (1 page) were created by reviewing package inserts, current commercial leaflets, and websites of pharmaceutical companies. The leaflet contained background information about: effects, indications, injection times, therapy durations, daily injections, potential adverse drug reactions of LMWH, potential interactions with over-the-counter (OTC) medication, actions to be taken if a dose was skipped, and thrombosis and embolism, including their symptoms and actions to be taken. The leaflet also included a step-by-step instruction in the s.c. injection technique with illustrations and explanations and a diary to record daily injections for self-monitoring. The manual was designed to be a quick reference card providing a brief summary of the sequential steps of the s.c. injection. Both the leaflet and manual were reviewed by a hematologist.

\subsection{Patient Recruitment and Randomisation}

In the clinical setting arm (RCT), patients received standard hospital care. Patient recruitment was performed by the primary investigator by regularly contacting the nurses or physicians to ask for potential study participants. The hospital staff had no further involvement in the study. If the patient met the inclusion criteria and written informed consent was obtained, a sharps collector (E-safe) for the used syringes and written patient information was delivered. The 1:1 randomisation was performed by using a research randomiser [21], and patients were sequentially assigned to the intervention or control group. Interventions were offered and applied according to patient need, either at the patient's bedside or immediately after discharge in the "Emergency Pharmacy Basel" - a community pharmacy open only at night, weekends, and holidays - which was used as study centre during the day. Patients of the control group (ClinS-C) received standard care by filling their prescription in the

Table 1. Range of possible interventions being offered to patients of the ClinS-I and DailyS-I arm.

\section{Intervention}

- Delivery of a leaflet (inclusive oral instruction of the leaflet: background information and step-by-step instruction in the s.c. injection technique)

- Delivery of a manual (brief summary of the sequential steps of an s.c. injection)

- Delivery of a kit (20 alcohol swabs, cotton swabs, and plasters)

- Oral instruction in s.c. injection technique

- Injection training into a "phantom” (injection pillow; PharmaDesign Inc., Warren, NJ, USA; delivered by Pfizer AG)

- Instruction in the injection technique using a commercial video (CD-ROM or website)

- (First) self-injection in the presence of a pharmacist (at patient's individual injection time)

- (First) injection administered by a pharmacist (ClinS-I: primary investigator; DailyS-I: trained community pharmacist)

ClinS-I: Intervention group, clinical setting; DailyS-I: Intervention group, daily life setting. 
community pharmacy of their choice.

In the daily life setting arm, routine prescription validation was performed by each community pharmacy when a LMWH was requested. If the patient met the inclusion criteria and informed consent was obtained, a sharps collector and written patient information was delivered. The trained community pharmacists of the DailyS-I offered and applied the interventions based on the SOP and according to each patient's needs. Patients of the control group received the community pharmacy's standard care.

\subsection{Self-Assessment (Patient Interviews)}

We used the same questionnaire as in a former study [14] for structured telephone interviews at the beginning and after completion of the s.c. therapy evaluating drug use problems, self-management, knowledge, quality of care, non-adherence rate, and patient satisfaction. The questionnaire was intensively tested for its comprehensibility and completeness.

To assess each patient's skills, we used 5-point Likert scales $(+2=$ correct answer; $+1 ; 0 ;-1 ;-2=$ wrong answer). To minimize bias generation with the complex data transformation, we completed missing data by the mean values. A committee consisting of a hematologist, a physician working as a medical advisor for Fragmin ${ }^{\circledR}$, a nurse, two clinical pharmacists, the primary investigator, and a master student involved in the study design rated the importance of the questions by using a 4-point scale. Cronbach's alpha reliability coefficient $(\alpha)$ was used as a measure of the internal consistency and reliability of the scores. The mean values on the 4-point scales (range: 1.6 - 3.9; $\alpha=0.80$ ) were converted into a weighting range between 1.0 and 2.0. This means that an item rated to be very important received twice as much emphasis as one rated to be of average importance. The scales were then computed as weighted means of the individual items (i.e. score minimum $=-2.00$; score maximum $=$ $+2.00)$. We defined different domains to group the questions and to facilitate comparisons. The domains were divided into "Self-assessment" (subjective by patients) and "Reality" (objective by investigators). They comprised assessments on application quality, assistance quality, adherence, and knowledge (Table 2). The catalogue with the questions assigned to the particular domains is listed in Appendix.

\subsection{Application Quality (D0T, Directly Observed Therapy)}

During a home visit and at her/his individual injection time, each patient was monitored when self-administering an s.c. injection. Patient's injection technique was objectively assessed by the investigator using a DOT-based data collection sheet (i.e. domain "Reality": application quality; Table 2, Appendix). A correlation test between self-assessed and observed application quality was used to analyse the necessity of the time- and cost-consuming DOT.

Table 2. Generation of 7 domains (each domain has a score range of score minimum $=-2.00$ and score maximum $=+2.00$ ).

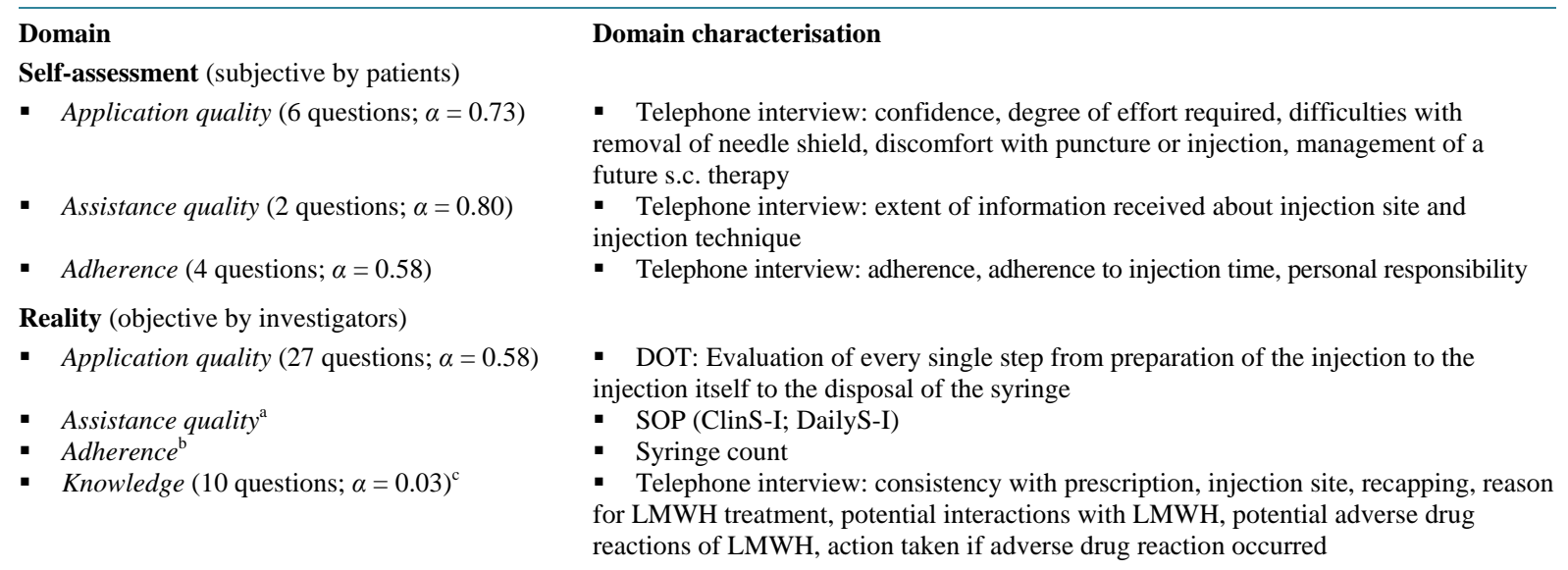

DOT directly observed therapy; SOP standard operating procedure; ClinS-I Intervention group, clinical setting; DailyS-I Intervention group, daily life setting; LMWH Low-molecular-weight heparins. a. No score: interventions were done only if required. b. No score: assessed using syringe count. c. Ceiling effect: nearly all patients were very knowledgeable about the treatment itself and inconsistently ignorant about questions of recapping, interactions with OTC medication, and adverse drug reactions. Scale consistency is low because while patients are consistently knowledgeable, they do not exhibit any consistent pattern regarding their (very limited) areas of ignorance. 
Data collection (telephone interviews and DOT) was performed by the primary investigator in the ClinS and DailyS-I arm. The DailyS-C arm consisted of a subpopulation of a former study [14], where trained master students had to recruit one patient and perform the interviews and the DOT. We standardised the questionnaire- and DOT-based data collection, approved the monitoring and recording skills of the students recruiting for the DailyS-C arm, and checked the primary investigator's and the students' consistency of rating (data not shown).

\subsection{Syringe Count}

The returned sharps collectors were examined for number of used syringes and number of syringes with recapping (illegitimate replacement of the needle shield after injection). By comparing the number of used syringes with the prescribed therapy duration, we determined levels of patient "taking" adherence (=syringe count). Missing syringes were used as a measure for non-adherence. If the therapy duration was not specified on the prescription or unclear (terms such as "treatment until complete mobilization, international normalized ratio (INR) twice in therapeutic range, next visit with physician”), we referred to the dates of the first and last injections. If these dates and the therapy duration were not provided, if there was an unscheduled visit with the physician/hospital, or if the sharps collectors were missing, we were unable to determine the adherence reliably and classified the patient's adherence as "not determinable". Patients who discontinued their s.c. treatment early due to full physical load were classified as fully compliant.

The study protocol was approved by the local Ethics Committee of Basel (EKBB 95/07; ClinicalTrials.gov identifier: NCT00794560).

\subsection{Statistical Analysis}

Data sheets were processed with the automated form-processing software TELEform ver. 10.2 (Cardiff Software, Vista CA). Plausibility testing was performed by the primary investigator. Because the statistical procedures used were either very straightforward, such as comparison of two sample means, or included parameters that were impossible to estimate with any confidence, such as the variance of questionnaire scores, we did not compute a power analysis. For a comparison of sample means, we have 32 - 40 subjects per group, so we can expect to find differences in the region of one half to one third of a standard deviation (SD). For our aim, which is to find differences that are relevant in daily life, we believe that this is sufficient. For more complex analyses, statistical power may vary, and will typically be somewhat higher, because the more complex statistical models are better at reducing error variation. Also, we use non-parametric comparisons if possible (Mann-Whitney test; for descriptive analysis: median and interquartile range [IQR: 25th to 75th percentile]), which are generally more stable, at a potential cost of statistical power. Note that for the domains we cite the arithmetic mean (and Student's $t$ test) and not the median. The median almost always takes the value of a scale level, so if the scale has three levels, the median can take only three different values. Because of the way we computed our scores, different scores have a different number of levels, rendering a comparison of score medians very difficult to interpret. Pearson's chi-square test was used to investigate possible associations between two variables in a four-fold table. Spearman's correlation coefficient $(r)$ was applied to test for correlations between domains. Statistical significance was set at $p \leq 0.05$. Statistical analysis was performed using SPSS for Windows ver. 20.0 (SPSS, Chicago, IL).

\section{Results}

\subsection{Setting and Patient Characteristics}

Of the 484 persons assessed for eligibility, 154 were included into the study and the data of 139 patients were analysed (Figure 1). Ten patients (16.7\%) out of 60 not meeting the inclusion criteria in the ClinS arm reported needle phobia. Fourteen community pharmacies recruited 1 - 7 patients, 7 community pharmacies could not recruit any patients. Patient and medication characteristics and parameters on patients' self-reports are summarised in Table 3. Patients of the ClinS arm were more experienced in self-injecting than patients of the DailyS arm ( $p$ $=0.04$ ). In the DailyS arm, patients of the control group mentioned less adverse drug reactions than patients of the intervention group $(p<0.01)$. This was confirmed by comparing the combined control (ClinS-C + DailyS-C) and intervention (ClinS-I + DailyS-I) groups $(p<0.01)$. Other patient characteristics and parameters on patient's 

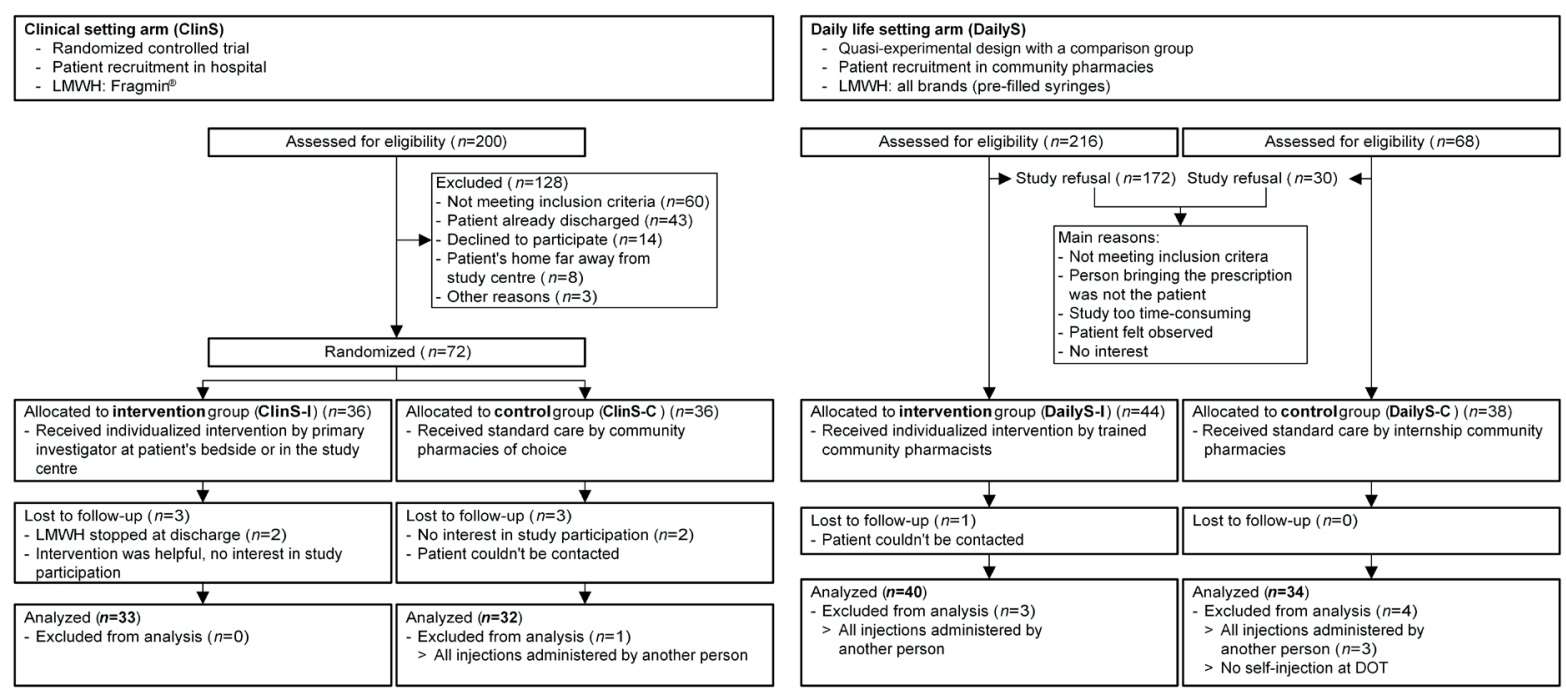

Figure 1. Study flowchart with reasons for exclusion. LMWH Low-molecular-weight heparins; DOT Directly observed therapy.

self-reports were comparable within the ClinS arm (ClinS-I vs. ClinS-C), within the DailyS arm (DailyS-I vs. DailyS-C), between the ClinS (ClinS-I + ClinS-C) and the DailyS (DailyS-I + DailyS-C), between the assembled intervention (ClinS-I + DailyS-I) and control (ClinS-C + DailyS-C) groups, and within the two intervention groups (ClinS-I vs. DailyS-I). No study participant had a thromboembolic event during the observation period (i.e. until the end of the individual LMWH treatment).

\subsection{Interventions}

According to our SOP, the objective assistance quality in the two study arms ClinS-I and DailyS-I was patient-tailored as interventions were offered and provided only if required (Table 4). No additional care was desired. Analysing the free-text comments of patients rating the interventions as not helpful, we worked out that these patients declared having no need of them (leaflet: $p<0.01$; manual: $p<0.01$; oral instructions: $p<0.01$; injection training into a "phantom": $p=0.01)$. Patients of the ClinS-I received more oral instructions (93.9\% vs. $70.0 \% ; p=0.02)$ and injection training into a "phantom" (84.8\% vs. 22.5\%; $p<0.01)$. On the other hand, patients of the DailyS-I assessed the leaflet to be more helpful (53.1\% vs. $80.6 \%$; $p=0.02)$.

\subsection{Application Quality (DOT) and Self-Assessment}

The DOT was performed 120 times at patients’ homes, 3 times at the study centre, 2 times at their workplace, and 3 patients recorded their injection for DOT on a video. We were unable to perform 11 DOTs (7.9\%) as: patients' individual therapy durations were very short and the treatment had already been terminated due to an INR in therapeutic range $(n=2)$ or full physical load $(n=2)$; the patient's home was too far away from study centre $(n=3)$; it was impossible to find an appropriate date $(n=2)$; or the patient refused the DOT $(n=2)$. The application quality was not influenced by age $(r=-0.10, p=0.15)$, sex $(p=0.63)$, previous outpatient s.c. injection therapies $(p=0.22)$, first self-injection in the presence of a medical professional $(p=0.38)$, fine motor skills $(p$ $=0.91$ ), or the injection site ( $p=0.06$; a trend towards a higher score with injections into the thigh (mean: 1.17 ; SD: +/ 0.34) than into the abdomen (mean: 1.03; SD: +/-0.39) was observed).

Table 5 shows the error rates of clinically relevant administration steps and the influence of the interventions upon them. Table 6 shows the scores of the patients in the different study arms, as well as for the combined intervention (ClinS-I + DailyS-I) and control (ClinS-C + DailyS-C) groups. There was no strong correlation between the domains $(r=-0.02$ to 0.20$)$. A direct correlation between "Reality" (objectively assessed by investigators, Table 2) and "Self-assessment” (subjectively assessed by patients themselves) was only possible for the application quality; it resulted in a low and non-significant correlation $(r=-0.10 ; p=0.20)$.

There were no score differences between the intervention groups of the ClinS and DailyS arms $(p \geq 0.14)$. 
Table 3. Characteristics of study sample $\left(n_{\text {total }}=139\right)$.

(a)

Age (years) (range: 18 - 84)

Male

Impairment in daily living due to arm, shoulder, or hand

Impaired vision (using glasses or contact lenses)

Medication:

$\begin{array}{ll}\text { - } & \text { Fragmin }^{\circledR} \text { (dalteparin) } \\ \text { - } & \text { Clexane }^{\circledR} \text { (enoxaparin) } \\ \text { - } & \text { Fraxiparine }^{\circledR} \text { (nadroparin) } \\ \text { - } & \text { Fraxiforte }^{\circledR} \text { (nadroparin) } \\ \text { - } & \text { Sandoparin }{ }^{\circledR} \text { (certoparin) } \\ & \text { Arixtra }{ }^{\circledR} \text { (fondaparinux) }\end{array}$

Application once daily:

- Not specified on prescription

Reason for LMWH treatment (multiple answers possible):

$\begin{array}{ll}\text { - } & \text { Injury/orthopedic surgery } \\ \text { - } & \text { Thrombosis, embolism } \\ \text { - } & \text { Perioperative management/bridging } \\ \text { - } & \text { Atrial fibrillation, myocardial infarction } \\ & \text { Other }\end{array}$

Previous outpatient s.c. injection therapies History of first self-injection in the presence of a medical professional

Injection site (multiple answers possible):

- Thigh

- Abdomen

Adverse drug reactions (multiple answers possible):

- Hematoma at injection site

- Mild injection site irritation/burning

- Hematoma in general

- Site pain

- Induration

- Exanthema

- Bleeding tendency; $n=1$ met criteria for reporting an adverse event to regulatory authority (melena)

- Epistaxis

- Other

Unscheduled visit with physician/hospital

Skipping injections:

- 1 time

- >3 times

Reason for skipping injections

(multiple answers possible):

- Forgotten

- Not being at home

- Early discontinuation

- Needle phobia

- Other

${ }^{*} p \leq 0.05$. a. All data are presented as the number of patients $(n)$ with the percentage in parenthesis, with the exception of "Age", which is presented as the median with the IQR in parenthesis. LMWH Low-molecular-weight heparins; s.c. Subcutaneous; ClinS-I Intervention group, clinical setting; ClinS-C Control group, clinical setting; DailyS-I Intervention group, daily life setting; DailyS-C Control group, daily life setting.

\begin{tabular}{|c|c|c|c|c|c|}
\hline \multirow[t]{6}{*}{$33(100.0)$} & $32(100.0)$ & $22(55.0)$ & $14(41.2)$ & $101(72.7)$ & $0(0.0)$ \\
\hline & & $11(27.5)$ & 5 (14.7) & $16(11.5)$ & \\
\hline & & $2(5.0)$ & 12 (35.3) & $14(10.1)$ & \\
\hline & & $1(2.5)$ & 2 (5.9) & $3(2.2)$ & \\
\hline & & $2(2.5)$ & $1(2.9)$ & $3(2.2)$ & \\
\hline & & $2(5.0)$ & $0(0.0)$ & $2(1.4)$ & \\
\hline 33 (100.0) & 30 (93.8) & 33 (82.5) & 27 (79.4) & 123 (88.5) & $2(1.4)$ \\
\hline \multirow[t]{2}{*}{$0(0.0)$} & $2(6.2)$ & 7 (17.5) & $4(11.8)$ & $13(9.4)$ & \\
\hline & & & & & $2(1.4)$ \\
\hline 31 (93.9) & 32 (100.0) & 31 (77.5) & 20 (58.8) & $114(82.0)$ & \\
\hline $2(6.1)$ & $0(0.0)$ & $3(7.5)$ & $3(8.8)$ & $8(5.8)$ & \\
\hline $0(0.0)$ & $0(0.0)$ & $4(10.0)$ & 2 (5.9) & $6(4.3)$ & \\
\hline $0(0.0)$ & $0(0.0)$ & $0(0.0)$ & 3 (8.8) & $3(2.2)$ & \\
\hline $0(0.0)$ & $0(0.0)$ & $3(7.5)$ & 4 (11.8) & $7(5.0)$ & \\
\hline $19(57.6)$ & 23 (71.9) & 19 (47.5) & 15 (44.1) & $76(54.7)$ & $2(1.4)$ \\
\hline \multirow[t]{2}{*}{$20(60.6)$} & $18(56.3)$ & 23 (57.5) & 20 (58.8) & $81(58.3)$ & $3(2.2)$ \\
\hline & & & & & $2(1.4)$ \\
\hline 27 (81.8) & $24(75.0)$ & $26(65.0)$ & 20 (58.8) & 97 (69.8) & \\
\hline $13(39.4)$ & 15 (46.9) & $18(45.0)$ & $16(47.1)$ & 62 (44.6) & \\
\hline 33 (100.0) & $29(90.6)$ & 37 (92.5) & $17(50.0)^{*}$ & 116 (83.5) & $2(1.4)$ \\
\hline 31 (93.9) & 26 (81.3) & 35 (87.5) & 15 (44.1) & 107 (77.0) & \\
\hline $16(48.5)$ & 17 (53.1) & $22(55.0)$ & 5 (14.7) & $60(43.2)$ & \\
\hline $2(6.1)$ & $2(6.3)$ & 5 (12.5) & $3(8.8)$ & 12 (8.6) & \\
\hline $3(9.1)$ & $0(0.0)$ & $4(10.0)$ & $3(8.8)$ & $10(7.2)$ & \\
\hline $5(15.2)$ & $0(0.0)$ & $3(7.5)$ & $0(0.0)$ & $8(5.8)$ & \\
\hline $1(3.0)$ & 2 (6.3) & $0(0.0)$ & $0(0.0)$ & $3(2.2)$ & \\
\hline $1(3.0)$ & $0(0.0)$ & $1(2.5)$ & $1(2.9)$ & $3(2.2)$ & \\
\hline $0(0.0)$ & $1(3.1)$ & $1(2.5)$ & $0(0.0)$ & $2(1.4)$ & \\
\hline $2(6.1)$ & $2(6.3)$ & $9(22.5)$ & $0(0.0)$ & $13(9.4)$ & \\
\hline $6(18.2)$ & $3(9.4)$ & 5 (12.5) & $0(0.0)$ & $14(10.1)$ & $1(0.7)$ \\
\hline $4(12.1)$ & $6(18.8)$ & 7 (17.5) & 1 (2.9) & 18 (12.9) & $9(6.5)$ \\
\hline $2 / 4(50.0)$ & 4/6 (66.7) & 6/7 (85.7) & $1^{\prime} 1(100.0)$ & $13 / 18(72.2)$ & $0 / 18(0.0)$ \\
\hline \multirow[t]{2}{*}{$0 / 4(0.0)$} & 1/6 (16.7) & 1/7 (14.3) & $0 / 1(0.0)$ & 2/18 (11.1) & \\
\hline & & & & & $0 / 18(0.0)$ \\
\hline $2 / 4(50.0)$ & 5/6 (83.3) & 5/7 (71.4) & $0 / 1(0.0)$ & $12 / 18$ (66.7) & \\
\hline $1 / 4(25.0)$ & 2/6 (33.3) & 1/7 (14.3) & $0 / 1(0.0)$ & 4/18 (22.2) & \\
\hline $1 / 4(25.0)$ & $0 / 6(0.0)$ & 1/7 (14.3) & $0 / 1(0.0)$ & 2/18 (11.1) & \\
\hline $0 / 4(0.0)$ & $0 / 6(0.0)$ & 1/7 (14.3) & $0 / 1(0.0)$ & 1/18 (5.6) & \\
\hline $2 / 4(50.0)$ & $0 / 6(0.0)$ & 2/7 (28.6) & $1^{\prime} 1(100.0)$ & 5/18 (27.8) & \\
\hline
\end{tabular}

$56(34-60) 56(42$ - 66) $51(36$ - 65) $54(43-67) 54(40-65) \quad 0(0.0)$

$17(51.5) \quad 13(40.6) \quad 23(57.5) \quad 16(47.1) \quad 69(49.6) \quad 0(0.0)$

$\begin{array}{llllll}(9.1) & 8(25.0) & 10(25.0) & 5(14.7) & 26(18.7) & 2(1.4)\end{array}$

$3(9.1) \quad 3(9.4) \quad 6(15.0) \quad 5(14.7) \quad 17(12.2) \quad 6(4.3)$

$\begin{array}{llllll}\text { ClinS-I } & \text { ClinS-C } & \text { DailyS-I } & \begin{array}{l}\text { DailyS-C } \\ (n=34)\end{array} & \begin{array}{l}\text { Total } \\ \left(n_{\text {total }}=139\right)\end{array} & \begin{array}{l}\text { Missing } \\ \text { data } \\ (n=32)\end{array} \\ n(\%)^{\mathrm{a}} & n(\%) & n(\%) & n(\%) & n(\%) & n(\%)\end{array}$


Table 4. Patients' assessment of the received assistance quality.

\begin{tabular}{|c|c|c|c|c|}
\hline & $\begin{array}{l}\text { ClinS-I } \\
(n=33)\end{array}$ & $\begin{array}{l}\text { DailyS-I } \\
(n=40)\end{array}$ & $\begin{array}{l}\text { ClinS-I + DailyS-I } \\
(n=73)\end{array}$ & Missing data \\
\hline & \multicolumn{4}{|c|}{ SOP, $n(\%)$} \\
\hline \multicolumn{5}{|l|}{ Assistance quality } \\
\hline - Delivery of leaflet-helpful & $17 / 32(53.1)^{*}$ & 29/36 (80.6) & $46 / 68(67.6)$ & $0 / 68(0.0)$ \\
\hline - Delivery of a manual-helpful & 20/32 (62.5) & $24 / 33(72.7)$ & $44 / 65(67.7)$ & $0 / 65(0.0)$ \\
\hline $\begin{array}{l}\text { - Delivery of a kit (alcohol/cotton swabs, } \\
\text { plasters)-helpful }\end{array}$ & 29/33 (87.9) & $38 / 39(97.4)$ & $67 / 72(93.1)$ & $0 / 72(0.0)$ \\
\hline - Delivery of a sharps collector-helpful & 22/33 (66.7) & $26 / 40(65.0)$ & $48 / 73(65.8)$ & 21/73 (28.8) \\
\hline - Oral instructions-helpful & 22/31 (71.0) & $23 / 28(82.1)$ & $45 / 59(76.3)$ & $0 / 59(0.0)$ \\
\hline - Injection training into a "phantom"-helpful & $21 / 28(75.0)$ & 8/9 (88.9) & 29/37 (78.4) & $0 / 37(0.0)$ \\
\hline - Commercial video tape-helpful & $0 / 0(0.0)$ & $0 / 0(0.0)$ & $0 / 0(0.0)$ & $0 / 0(0.0)$ \\
\hline $\begin{array}{l}\text { - (First) self-injection in the presence } \\
\text { of a pharmacist-helpful }\end{array}$ & $0 / 0(0.0)$ & $3 / 3(100.0)$ & $3 / 3(100.0)$ & $0 / 3(0.0)$ \\
\hline $\begin{array}{l}\text { - (First) injection administered by a } \\
\text { pharmacist-helpful }\end{array}$ & $1 / 1(100.0)$ & $0 / 0(0.0)$ & $1 / 1(100.0)$ & $0 / 1(0.0)$ \\
\hline
\end{tabular}

$* p \leq 0.05$. ClinS-I Intervention group, clinical setting; DailyS-I Intervention group, daily life setting.

Table 5. Error rates of clinically relevant administration steps and the influence of the interventions upon them.

\begin{tabular}{|c|c|c|c|}
\hline $\begin{array}{l}\text { s.c. injection steps (chronological listing) } \\
\text { Observations during the DOTs }(n=128)\end{array}$ & $n(\%)$ & $\begin{array}{l}\text { Missing data } \\
n(\%)\end{array}$ & $\begin{array}{l}\text { Intervention (ClinS-I + DailyS-I) vs. control } \\
(\text { ClinS-C + DailyS-C) } \\
\text { p value }\end{array}$ \\
\hline No washing or disinfection of hands right before injection & $85(66.4)$ & $2(1.6)$ & $p=0.01$ favouring intervention \\
\hline Not waiting for the alcohol to evaporate $(n=124)$ & $58(46.8)$ & $2(1.6)$ & $p=0.03$ favouring intervention \\
\hline Difficulties to remove needle shield & $12(9.4)$ & $0(0.0)$ & $p=0.84$ \\
\hline $\begin{array}{l}\text { Need of a new pre-filled syringe due to wrong removal of } \\
\text { needle shield }\end{array}$ & $1(0.8)$ & $2(1.6)$ & $p=0.29$ \\
\hline Removal of air bubble & $11(8.6)$ & $1(0.8)$ & $p=0.26$ \\
\hline Not pinching a skin fold & $15(11.7)$ & $0(0.0)$ & $p=0.84$ \\
\hline No puncture into cleansed skin area $(n=124)$ & $1(0.8)$ & $0(0.0)$ & $p=0.14$ \\
\hline Not inserted the full length of the needle into the skin & $13(10.2)$ & $0(0.0)$ & $p=0.29$ \\
\hline Not waited a second before withdrawing the needle & $43(33.6)$ & $0(0.0)$ & $p=0.19$ \\
\hline Skin fold released before withdrawing the needle $(n=117)$ & $12(10.3)$ & $1(0.9)$ & $p<0.01$ favouring intervention \\
\hline Recapping & $57(44.5)$ & $0(0.0)$ & $p<0.01$ favouring intervention \\
\hline $\begin{array}{l}\text { Syringe not disposed immediately after withdrawing the } \\
\text { needle }\end{array}$ & 45 (35.2) & $0(0.0)$ & $p=0.20$ \\
\hline
\end{tabular}

DOT directly observed therapy; ClinS-I Intervention group, clinical setting; ClinS-C Control group, clinical setting; DailyS-I Intervention group, daily life setting; DailyS-C Control group, daily life setting.

Previous outpatient s.c. injection therapies had no influence on the scores $(p \geq 0.16)$, but led to a decrease in subjective effort required to administer the injection $(p=0.02)$ and to an increase in confidence $(p=0.04)$. Patients who received oral instructions reached higher scores in the DOT $(p=0.04)$, but self-assessed their application quality more sceptically $(p=0.01)$. The delivery of a leaflet or manual and injection training into a "phantom" had no influence on the scores.

\subsection{Syringe Count}

The sharps collectors of 128 patients contained a total of 3137 syringes (median: 18, IQR: 10 - 39.5; range: 2 - 
Table 6. Summary of results for application quality, knowledge, self-assessment, and syringe count.

\begin{tabular}{|c|c|c|c|c|c|c|c|c|c|}
\hline & $\begin{array}{l}\text { ClinS-I } \\
(n=33)\end{array}$ & $\begin{array}{l}\text { ClinS-C } \\
(n=32)\end{array}$ & $p$ value & $\begin{array}{l}\text { DailyS-I } \\
(n=40)\end{array}$ & $\begin{array}{l}\text { DailyS-C } \\
(n=34)\end{array}$ & $p$ value & $\begin{array}{c}\text { ClinS-I + } \\
\text { DailyS-I } \\
(n=73)\end{array}$ & $\begin{array}{c}\text { ClinS-C + } \\
\text { DailyS-C } \\
(n=66)\end{array}$ & $p$ value \\
\hline Domains $^{\mathrm{a}}$ & \multicolumn{9}{|c|}{ Scores, mean (SD) } \\
\hline $\begin{array}{l}\text { Application quality } \\
\text { (DOT) }\end{array}$ & $1.25(0.27)$ & $0.86(0.33)$ & $p<0.01$ & $1.20(0.41)$ & $1.17(0.40)$ & $p=0.80$ & $1.22(0.36)$ & $1.02(0.39)$ & $p<0.01$ \\
\hline Knowledge & $1.10(0.38)$ & $0.95(0.41)$ & $p=0.05$ & $1.03(0.33)$ & $0.94(0.40)$ & $p=0.38$ & $1.06(0.35)$ & $0.95(0.41)$ & $p=0.03$ \\
\hline $\begin{array}{l}\text { Application quality, } \\
\text { self-assessment }\end{array}$ & $1.27(0.47)$ & $1.34(0.41)$ & $p=0.56$ & $1.20(0.53)$ & $1.20(0.38)$ & $p=0.97$ & $1.23(0.50)$ & $1.27(0.40)$ & $p=0.77$ \\
\hline $\begin{array}{c}\text { Adherence, } \\
\text { self-assessment }\end{array}$ & $1.43(0.58)$ & $1.32(0.78)$ & $p=0.93$ & $1.35(0.75)$ & $1.59(0.38)$ & $p=0.47$ & $1.38(0.68)$ & $1.46(0.62)$ & $p=0.68$ \\
\hline $\begin{array}{l}\text { Assistance quality, } \\
\text { self-assessment }\end{array}$ & $1.09(0.38)$ & $1.02(0.44)$ & $p=0.54$ & $1.19(0.68)$ & $1.52(0.62)$ & $p<0.01$ & $1.14(0.56)$ & $1.28(0.59)$ & $p=0.05$ \\
\hline \multirow[t]{2}{*}{$\begin{array}{c}\text { Adherence } \\
\text { (syringe count) }\end{array}$} & \multicolumn{9}{|c|}{$\%^{\mathrm{b}}$, mean (SD) } \\
\hline & $94.5(10.5)$ & $96.2(10.6)$ & $p=0.36$ & $95.1(10.0)$ & $97.5(4.2)$ & $p=0.72$ & $94.8(10.2)$ & $96.8(7.9)$ & $p=0.40$ \\
\hline
\end{tabular}

a. Score minimum $=-2.00$; score maximum $=+2.00$. b. Overall range $48 \%-100 \%$. ClinS-I Intervention group, clinical setting; ClinS-C Control group, clinical setting; DailyS-I Intervention group, daily life setting; DailyS-C Control group, daily life setting; DOT directly observed therapy.

93; Table 6). In 41.0\%, the therapy duration was not specified or unclear. The adherence of 24 (17.3\%) patients was not determinable; $12.9 \%$ of patients admitted skipping injections, whereas the objective syringe count detected non-adherence with $37.4 \%$ of patients $(p<0.01)$. A greater than 2-hour delay to the prescribed injection time was mentioned by $15.8 \%$ patients (4.3\% missing), leading to a correct "taking" adherence (syringe count), but to a non-adherence in terms of timing; there was no difference between the combined control (ClinS-C + DailyS-C) and intervention (ClinS-I + DailyS-I) groups ( $p=0.17)$.

\subsection{Hypotheses}

We confirmed our hypothesis that intensive pharmaceutical care resulted in more safety (improvement in application quality and knowledge, Table 6; reduction of error rates of clinically relevant administration steps, Table 5), but we had to reject our assumptions of improved adherence (self-report, Table 3; syringe count, Table 6), more satisfaction (self-assessment of assistance quality, Table 6), and fewer complications (self-reports on adverse drug reactions, Table 3).

\section{Discussion}

The rising prevalence of s.c. injection therapies with their potential for problems during self-administration and patient concerns provides an opportunity for community pharmacists to strengthen their role in the health care system. We developed an SOP for pharmacist interventions that was of good quality, adequate, appreciated, and feasible in daily life. The interventions resulted in improved application quality and knowledge, but there was no influence on adherence, satisfaction, or adverse drug reactions. Oral instructions had the highest impact. Our study lacked a strong impact due to the patients' already high baseline skills, making further improvement challenging. Clinical and daily life settings showed no differences.

\subsection{Setting and Patient Characteristics}

Patients of the ClinS arm were more experienced in self-injecting than patients of the DailyS arm. As this study, as well as our previous data [14] show that patients with experience have less discomfort and the injections require less effort, there might be some bias concerning the self-assessment of the application quality. The high prevalence of adverse drug reactions is eye-catching. Hematoma and mild injection site irritation/burning account for the majority. They are typical adverse drug reactions of s.c. injections and might be reduced, at least to 
some extent, by a slow injection [22]-[25].

\subsection{Interventions}

As the interventions of the SOP were not standardised, but patient-tailored, we did not focus on the time needed. The primary investigator estimated an average of $30 \mathrm{~min}$ was required for both recruitment and interventions, which is in line with the literature (10 - $45 \mathrm{~min})$ [18]-[20] [26]. No additional care was desired and patients had no need of the interventions if they rated them as unhelpful (e.g. previous s.c. injection therapies, medical professional, good patient care in the past). This illustrates that we provided an SOP for first instruction by a community pharmacist and subsequent pharmaceutical care during self-injection which was feasible in daily life, that the quality of our interventions was good and adequate, and that a single 2-hour course was sufficient. Patients in the ClinS received more oral instructions and injection training into a "phantom". This might explain why patients in the DailyS assessed the leaflet as being more helpful. Compared to our former study investigating a heterogeneous outpatient population receiving standard care [14], patients of the two intervention groups ClinS-I and DailyS-I received more leaflets and manuals, injection training into a "phantom", and (first) injection administered by or in the presence of a pharmacist.

\subsection{Application Quality (DOT) and Self-Assessment}

Regarding the score design, Cronbach's alpha reliability coefficients between 0.58 and 0.80 are acceptable. Removing the least reliable items from the scales did not increase scale reliabilities, indicating that the reliabilities reflect the scales as a whole and not an inconsistent item quality. The number of questions per domain varied (2 - 27 questions). This reflects that some domains were more complex in nature, and balancing the number of questions per domain would have led to a loss of information regarding these domains, as well as to lower reliability scores.

We saw no relevant correlations-neither between the single domains nor between the self-assessed and observed application quality. Therefore, an objective assessment of patient skills is crucial and makes the time- and cost-consuming DOT worthwhile.

Overall, patient baseline skills were high, with the lowest score being 0.86 on a range of -2.00 to +2.00 , making further improvement difficult. Oral instructions were the pivotal intervention of the SOP to improve the application quality. Although there were no significant differences between the scores of the intervention groups in the clinical and daily life settings, the results of the two settings are not comparable, as in the Dailys patients receiving interventions did not achieve better results in application quality and knowledge (as did patients of the ClinS-I).

A notable, though not intended result regards the control group's assessment of the assistance quality. Overall, the control group felt that they received a very good level of care, probably because researchers have contacted them several times (telephone interviews, home visit), inquired about their well-being, and generally showed an interest in them. This shows two things: first, that patient's assessment of assistance quality does not necessarily rely on the quality of pharmaceutical care they receive, and second that this assessment can be substantially improved by comparatively simple means, such as asking them how they are doing.

\subsection{Syringe Count}

We detected a higher objective (37.4\%) than subjective (12.9\%) non-adherence rate. Similar studies reported non-adherence rates of 4.3\% - 39.1\% [14] [16]-[20] [27]-[31]. Assuming that our patients were honest, they might not have disposed all syringes in their sharps collector, they might have interpreted a 10-day course as a 10-day postoperative and not 10-day ambulatory treatment, they might have administered 40 rather than 42 syringes in a 6-week course for practical reasons (=4 packages) [28], or the prescription of one package (= 10 syringes) did not necessarily mean that all of them had to be injected. It illustrates that the syringe count was not very reliable, though the only way to objectively determine non-adherence. The overall adherence rate was high with an overall mean of $95.8 \%$. Patients seem to have concerns with pre-filled syringes [14], but are aware of their need [28]. In further research, we propose investigating the relation between concerns and needs of those injecting LMWH (pre-filled syringes) and those taking new oral antithrombotic drugs (direct factor Xa or thrombin inhibitors), as with oral medication, much lower adherence rates of about 50\% are reported [32] [33]. 
Research on this field has started recently [34]-[36], but direct comparisons, especially on long-term treatments, are missing so far.

The strength of our study is the parallel implementation in community pharmacies, allowing: 1) investigation as to whether the interventions are feasible in daily life and 2) direct comparison of the results under controlled study conditions and daily life conditions. Nevertheless, one has to be aware that experimental conditions were more strictly controlled in the ClinS arm (RCT; recruitment and data collection by one person (primary investigator); few places of recruitment (four hospital wards); one LMWH brand) than in the DailyS arm (quasi-experimental design with a comparison group; recruitment and data collection by several persons (community pharmacists, master students, primary investigator); several places of recruitment (community pharmacies); all brands of LMWH). The main limitation of the study is the low overall consent rate of $31.8 \%$. In the ClinS, the main reasons for not meeting inclusion criteria were injections administered by another person, change of hospital ward, discharge when bridging was completed (oral anticoagulants in therapeutic range), no outpatient LMWH treatment, and comprehension difficulties due to language. The new oral anticoagulants [37] did not seem to account for a bias as they were only licensed in Switzerland in January 2009 or after completion of the study, respectively.

The results of our study allow important recommendations for daily practice, which are: 1) each person with a prescription for an outpatient LMWH treatment should be offered written information, alcohol swabs, a sharps collector, and oral instructions in s.c. injection technique, 2) the first self-injection should occur in the presence of a medical professional to ensure proper injection technique - if not done in the hospital, we encourage the pharmacists to be present (at patient's individual injection time) [11] [15] [38], 3) injection training into a "phantom" and further injections in the presence of or administered by a pharmacist are very supportive tools and should be applied if the patient requires lots of effort or has discomfort, and 4) potential needle phobia [39] and handling difficulties [14] should be kept in mind.

\section{Conclusion}

Our SOP was of good quality, adequate, appreciated, feasible in the daily life of community pharmacies, and resulted in improved application quality and knowledge, despite high baseline of patient skills. The home visits with the directly observed therapy were valuable in determining patient skills. Health care professionals should invest more time in delivering oral instructions-being the pivotal intervention in improving patients' application quality —and in injection training into a "phantom". Patients are capable of managing s.c. injection therapies in a satisfactory way and with high adherence if adequate assistance is provided.

\section{Acknowledgements}

We would like to thank all the involved physicians and nurses from the Kantonsspital Baselland, Bruderholz; the University Hospital Basel; and the Felix Platter-Spital, especially Markus L. Lampert, Michael Bodmer, Roland Bingisser, Norbert Suhm, Christian Ritter, and Petra Schroeder; the team of the "Emergency Pharmacy Basel"; and the participating community pharmacies for their effort and cooperation. We also thank the patients for participating, the students for data collection, Judith Kaiser and Raphaela von Gruenigen for their assistance in developing and performing the study, as well as Joëlle Bader (data sheets processing and analysis of used syringes), Philipp Walter (technical help), and Paul Lavender (English review) for their valuable contributions.

\section{Funding}

This study was supported by Pfizer AG through an unrestricted Investigator-Initiated Research Grant (IIRG).

\section{Conflict of Interests}

No conflicts of interest to declare.

\section{References}

[1] Guyatt, G.H., Akl, E.A., Crowther, M., Gutterman, D.D. and Schuunemann, H.J. (2012) Executive Summary: Antithrombotic Therapy and Prevention of Thrombosis, 9th Ed.: American College of Chest Physicians Evidence-Based Clinical Practice Guidelines. Chest, 141, 7S-47S. 
[2] Wiesner, C. (2001) Arzneimittelsicherheit in der Perihospitalphase_Eine patientenbezogene Analyse des Pharmakotherapieprozesses. Ph.D. Thesis, University of Basel, Basel.

[3] Hepler, C.D. and Strand, L.M. (1990) Opportunities and Responsibilities in Pharmaceutical Care. American Journal of Hospital Pharmacy, 47, 533-543.

[4] Frokjaer, B., Sondergaard, B. and Herborg, H. (2004) Evidence Report 3-Follow-Up on Outcomes of Drug Therapy (Pharmaceutical Care). Pharmakon, 1-16.

[5] Blenkinsopp, A. and Hassey, A. (2005) Effectiveness and Acceptability of Community Pharmacy-Based Interventions in Type 2 Diabetes: A Critical Review of Intervention Design, Pharmacist and Patient Perspectives. International Journal of Pharmacy Practice, 13, 231-240. http://dx.doi.org/10.1211/ijpp.13.4.0001

[6] Roughead, E.E., Semple, S.J. and Vitry, A.I. (2005) Pharmaceutical Care Services: A Systematic Review of Published Studies, 1990 to 2003, Examining Effectiveness in Improving Patient Outcomes. International Journal of Pharmacy Practice, 13, 53-70. http://dx.doi.org/10.1211/0022357055551

[7] Machado, M., Bajcar, J., Guzzo, G.C. and Einarson, T.R. (2007) Sensitivity of Patient Outcomes to Pharmacist Interventions. Part II: Systematic Review and Meta-Analysis in Hypertension Management. The Annals of Pharmacotherapy, 41, 1770-1781. http://dx.doi.org/10.1345/aph.1K311

[8] Machado, M., Bajcar, J., Guzzo, G.C. and Einarson, T.R. (2007) Sensitivity of Patient Outcomes to Pharmacist Interventions. Part I: Systematic Review and Meta-Analysis in Diabetes Management. The Annals of Pharmacotherapy, 41, 1569-1582. http://dx.doi.org/10.1345/aph.1K151

[9] Machado, M., Nassor, N., Bajcar, J.M., Guzzo, G.C. and Einarson, T.R. (2008) Sensitivity of Patient Outcomes to Pharmacist Interventions. Part III: Systematic Review and Meta-Analysis in Hyperlipidemia Management. The Annals of Pharmacotherapy, 42, 1195-1207. http://dx.doi.org/10.1345/aph.1K618

[10] Wubben, D.P. and Vivian, E.M. (2008) Effects of Pharmacist Outpatient Interventions on Adults with Diabetes Mellitus: A Systematic Review. Pharmacotherapy, 28, 421-436. http://dx.doi.org/10.1592/phco.28.4.421

[11] Miller, N.H., Hill, M., Kottke, T. and Ockene, I.S. (1997) The Multilevel Compliance Challenge: Recommendations for a Call to Action. A Statement for Healthcare Professionals. Circulation, 95, 1085-1090. http://dx.doi.org/10.1161/01.CIR.95.4.1085

[12] Haynes, R.B., Ackloo, E., Sahota, N., McDonald, H.P. and Yao, X. (2008) Interventions for Enhancing Medication Adherence. Cochrane Database of Systematic Reviews, 2, CD000011.

[13] Mengiardi, S., Goepfert, B., Tsakiris, D.A. and Hersberger, K.E. (2009) Pitfalls in Patient Self-Management of Subcutaneous Drug Application: Removal of Rubber Protection Caps from Ready-to-Use Syringes. European Journal of Clinical Pharmacology, 65, 1061-1062. http://dx.doi.org/10.1007/s00228-009-0681-0

[14] Mengiardi, S., Tsakiris, D.A., Lampert, M.L. and Hersberger, K.E. (2011) Drug Use Problems with Self-Injected Low-Molecular-Weight Heparins in Primary Care. European Journal of Clinical Pharmacology, 67, 109-120. http://dx.doi.org/10.1007/s00228-010-0956-5

[15] Karch, A.M. (2004) A Needling Problem. American Journal of Nursing, 104, 81-83. http://dx.doi.org/10.1097/00000446-200404000-00026

[16] Colwell, C.W., Pullido, P., Hardwick, M.E. and Morris, B.A. (2005) Patient Compliance with Outpatient Prophylaxis: An Observational Study. Orthopedics, 28, 143-147.

[17] Spahn, G. (2002) Compliance with Self-Administration of Heparin Injections in Outpatients. European Journal of Trauma and Emergency Surgery, 28, 104-109. http://dx.doi.org/10.1007/s00068-002-1176-1

[18] Watts, A.C., Howie, C.R. and Simpson, A.H. (2006) Assessment of a Self-Administration Protocol for Extended Subcutaneous Thromboprophylaxis in Lower Limb Arthroplasty. The Journal of Bone and Joint Surgery (British Volume), 88, 107-110. http://dx.doi.org/10.1302/0301-620X.88B1.17003

[19] Le Gall, C., Jacques, E., Medjebeur, C., Darques, L., Briand, F., Haddad, J., et al. (2006) Low Molecular Weight Heparin Self-Injection Training: Assessment of Feasibility, Tolerance and Economic Analysis in Emergency Departments. European Journal of Emergency Medicine, 13, 264-269. http://dx.doi.org/10.1097/00063110-200610000-00004

[20] Piazza, G., Nguyen, T.N., Morrison, R., Cios, D., Hohlfelder, B., Fanikos, J., et al. (2012) Patient Education Program for Venous Thromboembolism Prevention in Hospitalized Patients. The American Journal of Medicine, 125, 258-264. http://dx.doi.org/10.1016/j.amjmed.2011.09.012

[21] Urbaniak, G.C. and Plous, S. (2007) Research Randomizer (Version 3.0). Computer Software. http://www.randomizer.org/

[22] Zaybak, A. and Khorshid, L. (2008) A Study on the Effect of the Duration of Subcutaneous Heparin Injection on Bruising and Pain. Journal of Clinical Nursing, 17, 378-385.

[23] Chan, H. (2001) Effects of Injection Duration on Site-Pain Intensity and Bruising Associated with Subcutaneous Hepa- 
rin. Journal of Advanced Nursing, 35, 882-892. http://dx.doi.org/10.1046/j.1365-2648.2001.01925.x

[24] Balci Akpinar, R. and Celebioglu, A. (2008) Effect of Injection Duration on Bruising Associated with Subcutaneous Heparin: A Quasi-Experimental within-Subject Design. International Journal of Nursing Studies, 45, 812-817. http://dx.doi.org/10.1016/j.ijnurstu.2007.02.005

[25] Palese, A., Aidone, E., Dante, A. and Pea, F. (2013) Occurrence and Extent of Bruising According to Duration of Administration of Subcutaneous Low-Molecular-Weight Heparin: A Quasi-Experimental Case-Crossover Study. Journal of Cardiovascular Nursing, 28, 473-482. http://dx.doi.org/10.1097/JCN.0b013e3182578b87

[26] Harrison, L., McGinnis, J., Crowther, M., Ginsberg, J. and Hirsh, J. (1998) Assessment of Outpatient Treatment of Deep-Vein Thrombosis with Low-Molecular-Weight Heparin. Archives of Internal Medicine, 158, 2001-2003. http://dx.doi.org/10.1001/archinte.158.18.2001

[27] Deakin, D.E., Mishreki, A., Aslam, N. and Docker, C. (2010) Patient Compliance with Extended Low Molecular Weight Heparin Injections Following Hip and Knee Arthroplasty. Hip International, 20, 555-558.

[28] Rubenacker, S., Kaiser, J. and Guschmann, M. (2013) Compliance of Patients Undergoing Thromboprophylaxis with Enoxaparin: The Comfort Study. Der Chirurg, 84, 235-242.

[29] Karlinski, M., Stolarczyk, A., Siuda, M. and Ziolkowski, M. (2006) Compliance with Low Molecular Weight Heparin in Ambulatory Orthopedic Patients. Ortopedia Traumatologia Rehabilitacja, 8, 633-638.

[30] Bergqvist, D., Arcelus, J.I. and Felicissimo, P. (2012) Post-Discharge Compliance to Venous Thromboembolism Prophylaxis in High-Risk Orthopaedic Surgery: Results from the Ethos Registry. Thrombosis and Haemostasis, 107, 280-287. http://dx.doi.org/10.1160/TH11-07-0464

[31] Wilke, T., Moock, J., Muller, S., Pfannkuche, M. and Kurth, A. (2010) Nonadherence in Outpatient Thrombosis Prophylaxis with Low Molecular Weight Heparins after Major Orthopaedic Surgery. Clinical Orthopaedics and Related Research, 468, 2437-2453. http://dx.doi.org/10.1007/s11999-010-1306-8

[32] Sackett, D.L. and Snow, J.C. (1979) The Magnitude of Compliance and Non Compliance. In: Haynes, R.B., Taylor, D.W. and Sackett, D.L., Eds., Compliance in Health Care, Johns Hopkins University Press, Baltimore, 11-22.

[33] Horne, R. and Weinman, J. (1999) Patients’ Beliefs about Prescribed Medicines and Their Role in Adherence to Treatment in Chronic Physical Illness. Journal of Psychosomatic Research, 47, 555-567. http://dx.doi.org/10.1016/S0022-3999(99)00057-4

[34] Lebel, B., Malherbe, M., Gouzy, S., Parienti, J.J., Dutheil, J.J., Barrellier, M.T., et al. (2012) Oral Thromboprophylaxis Following Total Hip Replacement: The Issue of Compliance. Orthopaedics and Traumatology: Surgery and Research, 98, 186-192.

[35] Quante, M., Thate-Waschke, I. and Schofer, M. (2012) What Are the Reasons for Patient Preference? A Comparison between Oral and Subcutaneous Administration. Zeitschrift für Orthopädie und Unfallchirurgie, 150, 397-403.

[36] Wilke, T. (2009) Patient Preferences for an Oral Anticoagulant after Major Orthopedic Surgery: Results of a German Survey. Patient, 2, 39-49. http://dx.doi.org/10.2165/01312067-200902010-00005

[37] Weitz, J.I., Eikelboom, J.W. and Samama, M.M. (2012) New Antithrombotic Drugs: Antithrombotic Therapy and Prevention of Thrombosis, 9th Ed.: American College of Chest Physicians Evidence-Based Clinical Practice Guidelines. Chest, 141, e120S-e151S.

[38] Treasure, T. (2011) Venous Thromboembolism—Reducing the Risk. NICE Guideline. http://www.nice.org.uk/guidance/cg92

[39] Turner, A.P., Williams, R.M., Sloan, A.P. and Haselkorn, J.K. (2009) Injection Anxiety Remains a Long-Term Barrier to Medication Adherence in Multiple Sclerosis. Rehabilitation Psychology, 54, 116-121. http://dx.doi.org/10.1037/a0014460 


\section{Appendix}

Questions assigned to the particular domains.

\begin{tabular}{|c|c|}
\hline $\begin{array}{l}\text { Domains: } \\
\text { Self-assessment } \\
\text { (subjective by patients) }\end{array}$ & 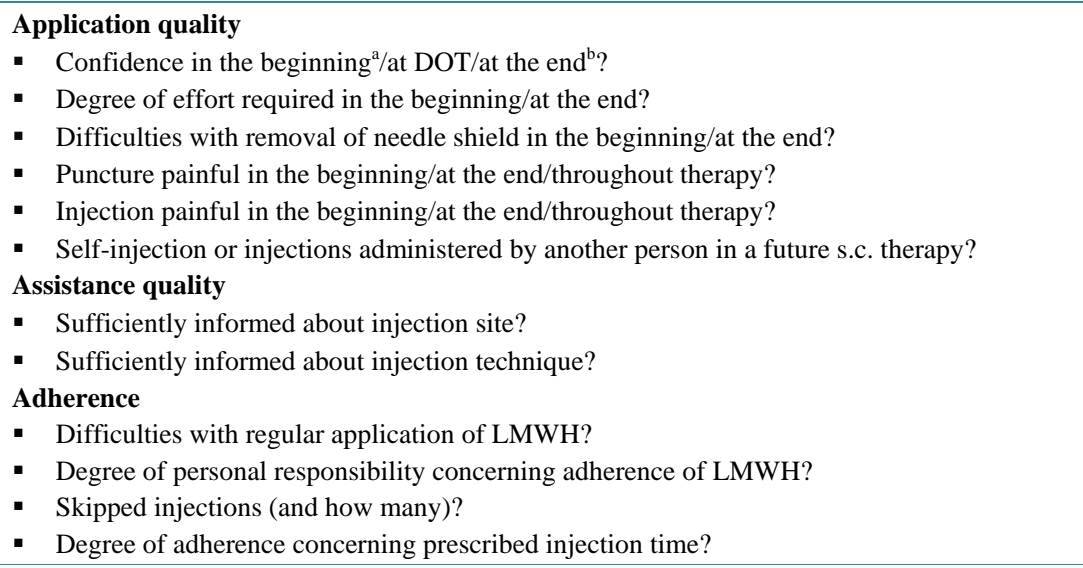 \\
\hline $\begin{array}{l}\text { Domains: } \\
\text { Reality } \\
\text { (objective by investigators) }\end{array}$ & 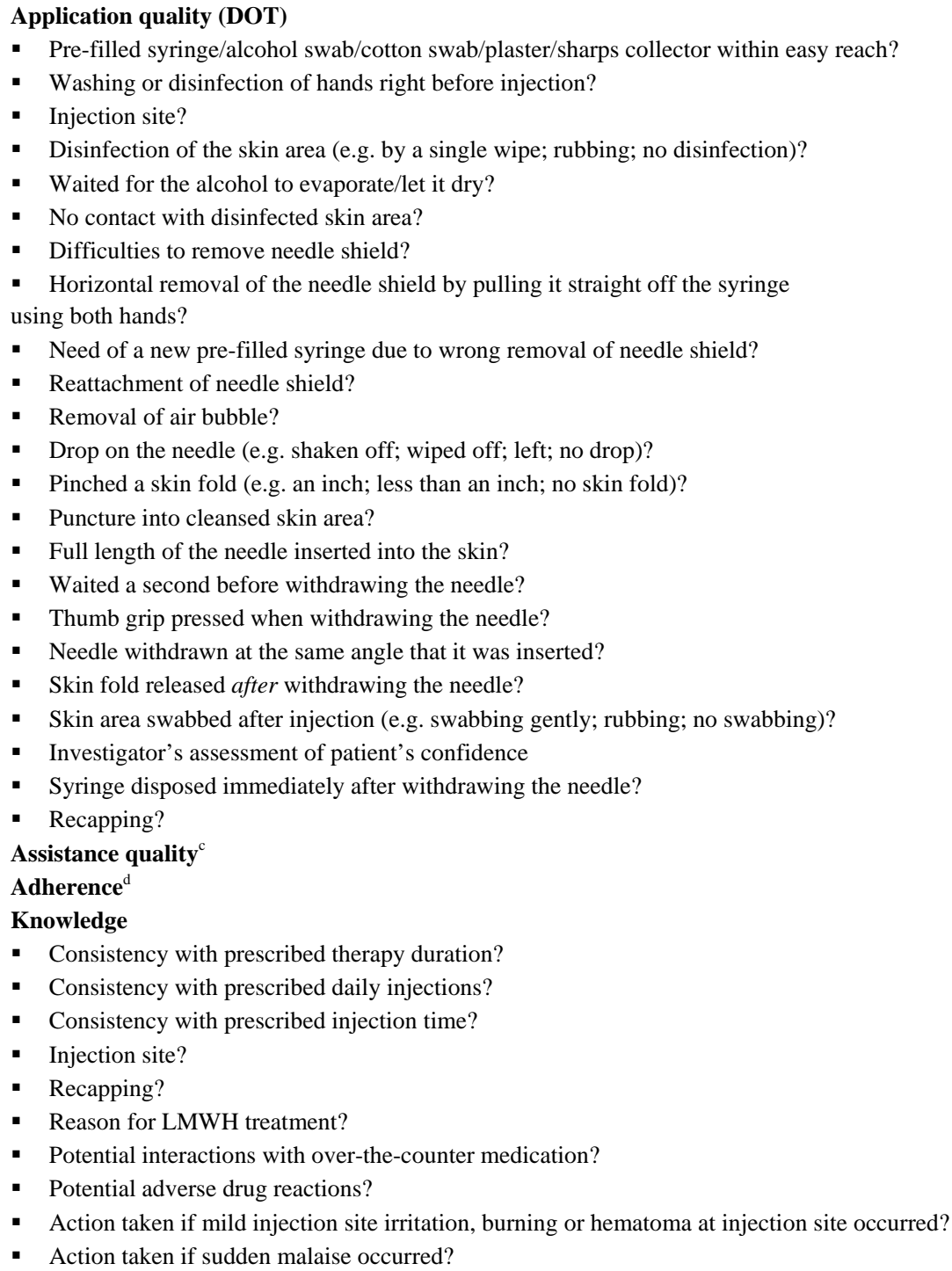 \\
\hline
\end{tabular}

a. Asked for at telephone interview. b. Asked for at final interview. c. No score: interventions were carried out only if required. d. No score: syringe count used. 